\title{
UM ENFOQUE PROBABILÍSTICO PARA A AVALIAÇÃO DO AMBIENTE INTERFERENTE ENVOLVENDO SATÉLITES GEOESTACIONÁRIOS
}

\author{
José Mauro P. Fortes e Raimundo Sampaio Neto
}

\begin{abstract}
Resumo - Este artigo propõe um enfoque probabilístico para a análise do ambiente interferente envolvendo satélites geoestacinários visando à elaboração de diagnósticos de arcos orbitais. Estes diagnósticos permitem a avaliação do potencial de cada posição orbital quanto à sua localização no arco orbital e às dificuldades de coordenação entre redes de comunicação por satélite (processo de negociação bilateral para solucionar eventuais problemas de interferência). $\mathrm{O}$ enfoque proposto utiliza métricas que refletem tanto a necessidade quanto a dificuldade esperada no processo de coordenação e permite uma avaliação do congestionamento orbital com um esforço computacional muito inferior ao requerido por uma análise determinística detalhada. Exemplos numéricos ilustrando a aplicação da metodologia proposta à situações práticas são apresentadas.
\end{abstract}

Palavras-chave: órbita de satélites geoestacionários, análise de interferências, enfoque probabilístico, diagnóstico de ocupação orbital

\begin{abstract}
This paper proposes a probabilistic new approach to satellite interference analysis for the assessment of the geostationary-satellite orbit utilization. The analysis allows for the evaluation of the potential of any given orbital position in terms of both the need for and the difficulty of network coordination (bilateral negotiation aiming at the solution of eventual interference problems). Appropriate metrics, that reflect both aspects of the coordination process (need and expected difficulty) are defined and used in the proposed methodology. The probabilistic approach provides an effective evaluation of the GSO use and requires much less computer effort than those required by usual deterministic approaches. Numerical examples involving practical situations are presented and discussed.
\end{abstract}

Keywords: geoestacionary satellite orbit, interference analysis, probabilistic approach, orbital arc evaluation

\section{INTRODUÇÃO}

A utilização de satélites em sistemas de comunicações proporcionou um grande crescimento na área de comunicações

Este trabalho teve suporte financeiro da Agência Nacional de Telecomunicações - Anatel através do Contrato PVSS-045/2001. José Mauro Fortes (jmfortes@cetuc.puc-rio.br) e Raimundo Sampaio Neto (raimundo@cetuc.puc-rio.br) são professores do Centro de Estudos em Telecomunicações da Pontifícia Universidade Católica do Rio de Janeiro. de longa distância. A possibilidade de se utilizar satélites geoestacionários, evitando dinâmicas relativas entre os transmissores e receptores, foi um fator determinante no desenvolvimento da área, iniciado na década de 60. Atualmente o número de sistemas de comunicações notificados junto à União Internacional de Telecomunicações (UIT) que utilizam satélites geoestacionários ultrapassa a casa dos 2000. A órbita em torno da Terra que permite a geoestacionariedade dos satélites é única e corresponde a uma órbita circular, no plano do equador, a uma altura de aproximadamente 36.000 kilometros. Assim, torna-se importante a utilização eficiente da órbita de satélites geoestacionários - OSG, de modo a garantir que um maior número de satélites possa compartilhar este recurso limitado mantendo um ambiente de interferência mútua aceitável para o desempenho adequado dos sistemas. A responsabilidade desta garantia cabe à UIT que, ao receber uma notificação propondo um novo sistema, procede a uma análise de interferência que determina o potencial das interferencias produzida e sofrida pelo novo sistema, autorizando ou não a sua operação, dependendo dos resultados desta análise. Eventuais problemas de interferências inadequadas são comunicados às partes interessadas que devem, por meio de negociações bilaterais (processo de coordenação), chegar a um acordo que viabilize tecnicamente a operação dos sistemas.

Assim, ao se notificar um novo sistema é importante que se tenha uma avaliação preliminar do ambiente interferente envolvendo os sistemas notificados que possuem satélites (em operação ou já autorizados pela UIT) na OSG de modo a identificar as posições orbitais mais adequadas para o satélite proposto, ou seja, se disponha de um diagnóstico atualizado da utilização da OSG. Devido às constantes alterações do ambiente interferente (sistemas novos são notificados e sistemas mais antigos saem de operação), esta avaliação deve ser feita periodicamente. Entretanto, dada a grande variedade e quantidade de parâmetros envolvidos, uma análise detalhada deste ambiente é bastante complexa e requer um alto esforço computacional. A impossibilidade da UIT de efetuar os cálculos necessários em tempo hábil, por exemplo, tem resultado em uma fila de sistemas notificados que esperam pela análise oficial da UIT. Num passado recente esta fila correspondia a um atraso de aproximadamente 3 anos. Atualmente, medidas adotadas pela UIT reduziram este atraso para 6 meses. Como alternativa à uma análise detalhada, este artigo apresenta um enfoque probabilístico para a avaliação do ambiente interferente envolvendo satélites geoestacinários visando a elaboração de diagnósticos de arcos orbitais.

Um diagnóstico de utilização do arco orbital deve refletir o grau de congestionamento das diversas posições na 
OSG, permitindo avaliar o potencial de cada posição orbital quanto a sua localização no arco orbital e as dificuldades de coordenação. O grau de congestionamento de uma dada posição orbital deve, por sua vez, refletir a quantidade de interferência que o satélite de um determinado sistema experimenta ou produz nos demais sistemas quando localizado na posição orbital considerada. No presente trabalho a medida da quantidade de interferência existente entre dois sistemas é baseada na, assim chamada, razão portadora-interferência co-canal generalizada.

Em uma análise de interferência baseada em cálculos de interferência de entrada única (single-entry), um critério usual é o de verificar, para cada um dos possíveis pares de sistemas, se o valor da razão portadora-interferência generalizada de single-entry associado a cada um dos sistemas está ou não acima de um limite pré-especificado (correspondendo a um valor positivo de margem) que caracterize uma condição de interferência aceitável entre as duas redes de cada par. Destas comparações obtem-se uma indicação da possibilidade de convivência entre as redes consideradas.

Como, nas situações consideradas, tanto o sistema interferente quanto o sistema interferido possuem vários enlaces, onde cada par enlace-interferente/enlace-interferido apresenta um valor de razão portadora-interferência co-canal generalizada, um possível enfoque seria o de tomar-se como referência o pior caso de razão portadora-interferência entre o par de sistemas. Observa-se, entretanto, que o pior caso de razão portadora-interferência generalizada tende a ocorrer com uma probabilidade muito pequena e, portanto, a sua adoção como figura de mérito pode ser muito restritiva para objetivo de se obter um diagnóstico de utilização do arco orbital.

Considerando este aspecto, é proposto e adotado aqui um enfoque probabilístico, no qual o valor da razão portadorainterferência generalizada associada a um dado par de sistemas foi modelada como uma variável aleatória cujo valor é função de cada possível par de enlaces interferenteinterferido considerado. Quanto maior for a probabilidade desta variável aleatória exceder um valor de limiar préespecificado que caracterize uma condição de interferência aceitável (probabilidade de ocorrência de margens positivas) menos restritivas deverão ser as condições de convivência entre os dois sistemas considerados.

Assim, com base neste enfoque probabilístico são introduzidas neste artigo um conjunto de figuras de mérito representadas por métricas que permitem avaliar a necessidade e a dificuldade de coordenação entre pares de sistemas. A partir destas métricas, são definidas outras métricas voltadas para a avaliação da necessidade e dificuldade de coordenação quando múltiplos sistemas são considerados. Este conjunto de métricas, foi utilizado em exemplos de elaboração de diagnósticos de utilização de arcos orbitais especíicos.

A Seção 2 deste documento apresenta as expressões utilizadas no cálculo das razões portadora-interferência generalizada co-canal de entrada única. O enfoque probabilístico aqui proposto, que trata estas razões portadora-interferência como variáveis aleatórias, assim como o procedimento proposto para a determinação da caracterização estatística dessas variáveis aleatórias é escrito nesta Seção 3. Ainda nesta seção são definidas as figuras de mérito, que conforme já mencionado, foram utilizadas na elaboração de exemplos de diagnósticos de utilização de arcos orbitais.

A Seção 4 apresenta exemplos de aplicação do enfoque probabilístico. Na Subseção 4.1 são destacados alguns aspectos dos procedimentos de cálculo levados a efeito na implementação da metodologia para a elaboração de diagnósticos proposta neste trabalho. Estes aspectos incluem o pré-processamento dos dados disponíveis no banco de dados da UIT, visando a redução do tempo de computação requerido para a produção dos resultados, e o posicionamento geográfico das estações terrenas envolvidas nos cálculos de interferência, que inclui o procedimento adotado para a identificação das posições mais desfavoráveis das estações transmissora-interferente e receptora interferida.

Nas Seções 4.2 e 4.3 as figuras de mérito (métricas) introduzidas neste artigo são utilizadas em exemplos que ilustram a avaliação de uma dada posição orbital bem como o diagnóstico da ocupação de um arco orbital específico em torno desta posição orbital. Comentários e conclusões são apresentados na Seção 5.

\section{ANÁLISE DE INTERFERÊNCIA}

Nesta seção são apresentadas as expressões das razões portadora-interferência generalizada co-canal de entrada única (single-entry) correspondentes ao lance de subida, ao lance de descida e ao enlace total .

\subsection{RAZÃO PORTADORA-INTERFERÊNCIA SINGLE-ENTRY}

Nesta seção considera-se a situação de interferência de entrada única (single-entry) apresentada na Figura 1 onde estão ilustrados dois enlaces de comunicação por satélite: um deles pertencente à rede interferente, que utiliza o satélite $S_{I}$, e o outro pertencente à rede interferida, que utiliza o satélite $S_{V}$. Note que os parâmetros associados ao enlace da rede interferente estão indicados por letras assinaladas superiormente com o sinal “"”. Considerando esta figura, a razão portadorainterferência nos terminais da antena de recepção do satélite $S_{V}$ (razão portadora-interferência no lance de subida) se escreve

$$
\left(\frac{C}{I}\right)_{u p}=\frac{\frac{P_{1} g_{1}(0) g_{2}(\phi)}{\ell_{s u}}}{\frac{P_{1}^{\prime} g_{1}^{\prime}(\theta) g_{2}(\rho)}{\ell_{s u^{\prime}}}}=\frac{P_{1} g_{1}(0) g_{2}(\phi) \ell_{s u^{\prime}}}{P_{1}^{\prime} g_{1}^{\prime}(\theta) g_{2}(\rho) \ell_{s u}}
$$

onde $P_{1}$ e $P_{1}^{\prime}$ representam respectivamente as potências nos terminais das antenas das estações terrenas transmissoras das redes interferida e interferente, $\ell_{s u}$ é a perda de espaço livre associada ao percurso do sinal desejado no lance de subida e $\ell_{s u^{\prime}}$ é a perda de espaço livre associada ao percurso da interferência no lance de subida. Os ganhos de antenas que aparecem em (1) correspondem a:

$g_{1}^{\prime}(\theta)$ ganho da antena da estação terrena transmissora interferente numa direção que forma um ângulo $\theta$ com a direção de apontamento da antena; 


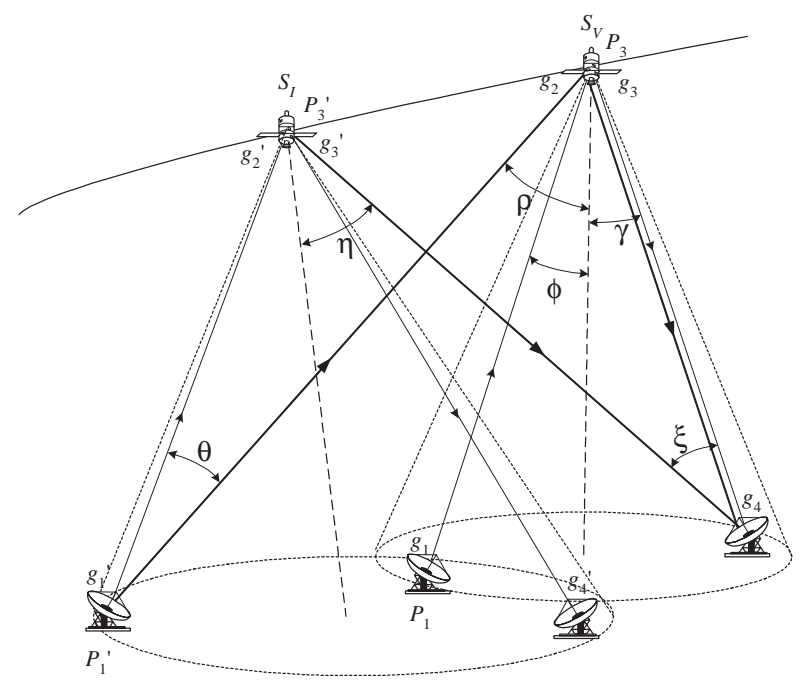

Figura 1. Cálculo de interferência: geometria do problema

$g_{1}(0)$ ganho máximo da antena da estação terrena transmissora interferida;

$g_{2}(\phi)$ ganho da antena receptora do satélite interferido numa direção que forma um ângulo $\phi$ com a direção de apontamento do feixe;

$g_{2}(0)$ ganho máximo da antena receptora do satélite interferido.

A razão portadora-interferência nos terminais da antena da estação terrena receptora da rede interferida (razão portadorainterferência no lance de descida) se escreve

$$
\left(\frac{C}{I}\right)_{\text {down }}=\frac{\frac{P_{3} g_{3}(\gamma) g_{4}(0)}{\ell_{s d}}}{\frac{P_{3}^{\prime} g_{3}^{\prime}(\eta) g_{4}(\xi)}{\ell_{s d^{\prime}}}}=\frac{P_{3} g_{3}(\gamma) g_{4}(0) \ell_{s d^{\prime}}}{P_{3}^{\prime} g_{3}^{\prime}(\eta) g_{4}(\xi) \ell_{s d}}
$$

onde $P_{3}$ e $P_{3}^{\prime}$ representam respectivamente as potências nos terminais das antenas transmissoras dos satélites das redes interferida e interferente, $\ell_{s d}$ é a perda de espaço livre associada ao percurso do sinal desejado, no lance de descida e $\ell_{s d^{\prime}}$ é a perda de espaço livre associada ao percurso da interferência no lance de descida. Os ganhos de antenas que aparecem em (2) correspondem a:

$g_{3}^{\prime}(\eta)$ ganho da antena transmissora do satélite interferente numa direção que forma um ângulo $\eta$ com a direção de apontamento do feixe;

$g_{3}(\gamma)$ ganho da antena transmissora do satélite interferido numa direção que forma um ângulo $\eta$ com a direção de apontamento do feixe;

$g_{4}(\xi)$ ganho da antena da estação terrena receptora interferida numa direção que forma um ângulo $\xi$ com a direção de apontamento da antena;

$g_{4}(0)$ ganho máximo da antena da estação terrena receptora interferida.

A partir de (1) e (2) obtém-se a razão portadora/interferência-total nos terminais da antena da estação terrena receptora da rede interferida, dada por

$$
\begin{aligned}
\left(\frac{C}{I}\right)_{\text {tot }} & =\left[\left(\frac{C}{I}\right)_{u p}^{-1}+\left(\frac{C}{I}\right)_{\text {down }}^{-1}\right]^{-1} \\
& =\left[\frac{P_{1}^{\prime} g_{1}^{\prime}(\theta) g_{2}(\rho) \ell_{s u}}{P_{1} g_{1}(0) g_{2}(\phi) \ell_{s u^{\prime}}}+\frac{P_{3}^{\prime} g_{3}^{\prime}(\eta) g_{4}(\xi) \ell_{s d}}{P_{3} g_{3}(\gamma) g_{4}(0) \ell_{s d^{\prime}}}\right]^{-1}
\end{aligned}
$$

Embora a expressão em (3) forneça a razão entre as potências da portadora desejada e da interferência total (lances de subida e descida), uma medida mais adequada é dada pela razão entre a potência da portadora desejada e a potência interferente contida na faixa de freqüências ocupada por esta portadora. Supondo-se que a emissão interferente ocupa toda a faixa da portadora desejada (uma única portadora interferente ou várias portadoras interferentes idênticas), esta razão se escreve

$$
\left(\frac{\mathcal{C}}{\mathcal{I}}\right)_{t o t}=\left(\frac{\frac{C}{b}}{\frac{I}{b^{\prime}}}\right)_{t o t}=\left[\frac{p_{1}^{\prime} g_{1}^{\prime}(\theta) g_{2}(\rho) \ell_{s u}}{p_{1} g_{1}(0) g_{2}(\phi) \ell_{s u^{\prime}}}+\frac{p_{3}^{\prime} g_{3}^{\prime}(\eta) g_{4}(\xi) \ell_{s d}}{p_{3} g_{3}(\gamma) g_{4}(0) \ell_{s d^{\prime}}}\right]^{-1}
$$

onde $b^{\prime}$ representa a banda ocupada pela portadora interferente, $b$ representa a banda ocupada pela portadora interferida e

$$
\begin{aligned}
p_{1} & =\frac{P_{1}}{b} \\
p_{3} & =\frac{P_{3}}{b} \\
p_{1}^{\prime} & =\frac{P_{1}^{\prime}}{b^{\prime}} \\
p_{3}^{\prime} & =\frac{P_{3}^{\prime}}{b^{\prime}}
\end{aligned}
$$

Note que, mesmo que a emissão interferente seja constituída por várias portadoras idênticas ocupando a faixa da portadora desejada, a quantidade $I$ em (4) representa a potência interferente associada a uma única destas portadoras. A razão em (4) é usualmente conhecida como razão portadorainterferência generalizada.

$\mathrm{Na}$ base de dados oficial da UIT [6] a quantidade de dados associada a cada rede de comunicações por satélite é extensa. Além de conter informações básicas sobre as características do sistema (e.g. posição orbital, potências, diagramas de radiação das antenas, temperaturas de ruído, etc.) estes dados contêm informações sobre o conjunto de portadoras que cada rede pretende transmitir. Usualmente os dados de uma dada rede de comunicações por satélite são estruturados da seguinte maneira: Considere um determinado satélite em uma dada posição orbital. Este satélite possui diversos feixes (feixes de transmissão e feixes de recepção). Para cada um deles existe um conjunto das portadoras ou emissões que poderiam ser transmitidas (feixe de transmissão) ou recebidas (feixe de recepção) pelo feixe. Associada a cada uma dessas portadoras existe um conjunto de parâmetros relativos aos enlaces que utilizam a portadora (e.g. características das estações terrenas, potências, dados de propagação, etc.). Além disso, existe ainda informação das possíveis ligações entre tranponders e feixes de antenas no satélite (transponder strapping). Com base nessas informações, é possível criar o 
conjunto de todos os possíveis enlaces associados a um determinado sistema (ver Seção 4.1.1). Uma vez identificados os conjuntos de enlaces associados aos sistemas interferente e interferido, e especificadas as posições das estações transmissora e receptora de cada enlace, é possível calcular, utilizando (4), o valor da razão portadora-interferência generalizada associada aos possíveis pares de enlace (enlace interferente,enlace interferido).

Considere um ambiente interferente contendo $M$ redes de comunicação por satélite. Seja $i,(i=1, \ldots, M)$ o índice que caracteriza a rede interferida e $j,(j=1, \ldots, M ; j \neq i)$ o índice que caracteriza a rede interferente. Sejam $\mathcal{E}_{i}$ e $\mathcal{E}_{j}$ os conjuntos de possíveis enlaces associados respectivamente à rede interferida $i$ e à rede interferente $j$. Considere a situação específica ilustrada na Figura 2, onde se deseja avaliar a interferência co-canal produzida pelo enlace $\ell\left(\ell \in \mathcal{E}_{j}\right)$ da rede interferente $j$ no enlace $k\left(k \in \mathcal{E}_{i}\right)$ da rede interferida $i$. Esta avaliação requer o conhecimento das posições geográficas das estações terrenas transmissora-interferente e receptora-interferida. Supõe-se que a primeira delas esteja localizada na posição geográfica mais desfavorável (correspondente ao maior nível de interferência de up-link) e a segunda esteja também localizada na posição mais desfavorável (correspondente ao maior nível de interferência de down-link). A determinação destas posições mais desfavoráveis é feita de acordo com o procedimento descrito na Seção 4.1.2). Nestas condições, utilizando-se (4) é possível determinar a razão portadora/interferência-total correspondente ao maior nível de interferência produzido pelo enlace $\ell$ da rede $j$ no enlace $k$ da rede $i$. Esta razão é aqui denotada por

$$
\left(\frac{\mathcal{C}}{\mathcal{I}}\right)_{i, k}^{j, \ell}
$$

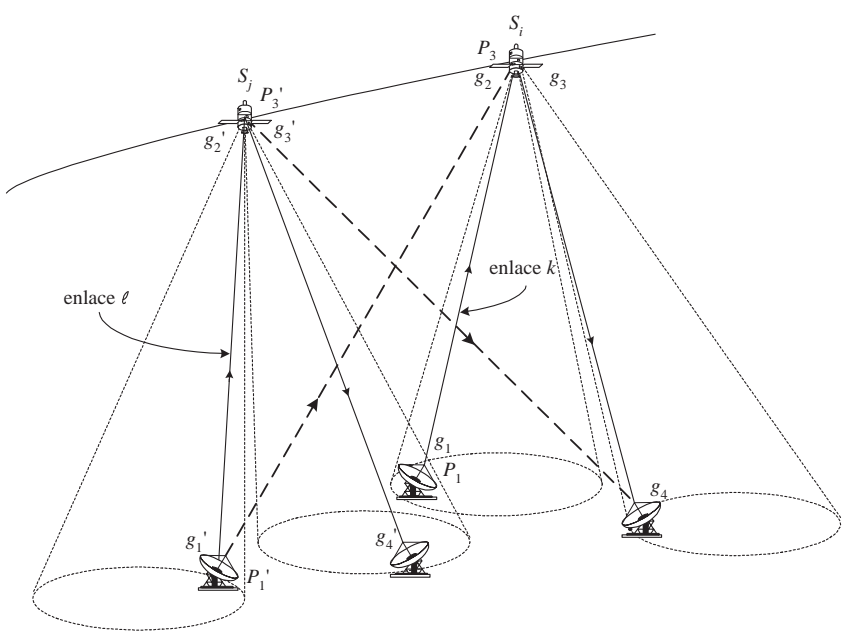

Figura 2. Enlaces dos sistemas interferente e interferido

Observe que o conhecimento de $(\mathcal{C} / \mathcal{I})_{i, k}^{j, \ell}$ para quaisquer valores de $i, j, k$ e $\ell$ permite uma grande flexibilidade na análise a ser feita. A partir desses valores é possível, por exemplo, calcular a razão portadora/interferência-total $(\mathcal{C} / \mathcal{I})_{i}^{j}$ correspondente ao efeito do enlace mais interferente da rede $j$ no enlace mais sensível da rede $i$ considerando que as estações terrenas interferente e interferida estão localizadas nas posições geográficas mais desfavoráveis. Esta razão portadora/interferência-total é dada por

$$
\left(\frac{\mathcal{C}}{\mathcal{I}}\right)_{i}^{j}=\min _{k \in \mathcal{E}_{i}}\left[\min _{\substack{\ell \\ \ell \in \mathcal{E}_{j}}}\left(\frac{\mathcal{C}}{\mathcal{I}}\right)^{j, \ell}\right]
$$

e constitui o pior caso de interferência produzido por um enlace da rede $j$ num enlace da rede $i$.

Em uma análise de interferência baseada em cálculos de interferência de single-entry, um critério usual é o de verificar, para cada um dos possíveis pares de sistemas, se o valor da razão $(\mathcal{C} / \mathcal{I})$ generalizada de single-entry associado a cada um dos sistemas está ou não acima de um limite $\eta$ préespecificado que caracterize uma condição de interferência aceitável entre as duas redes de cada par. Destas comparações obtem-se uma indicação da possibilidade de convivência entre as redes consideradas.

Observe entretanto que o pior caso de $(\mathcal{C} / \mathcal{I})$ generalizado, obtido por meio de (9), tende a ocorrer com uma probabilidade muito pequena e, portanto, a sua adoção como figura de mérito pode ser muito restritiva para o nosso objetivo de obter um diagnóstico de utilização do arco orbital. Uma alternativa mais adequada seria a utilização de um enfoque probabilístico no tratamento das quantidades envolvidas. Isto permitiria determinar, por exemplo, para cada um dos possíveis pares de sistemas, a probabilidade da razão $(\mathcal{C} / \mathcal{I})$ generalizada de single-entry associada a cada sistema do par exceder o limite $\eta$, possibilitando assim uma melhor avaliação do congestionamento do arco orbital. Este enfoque probabilístico é tratado na seção a seguir.

\section{ENFOQUE PROBABILÍSTICO PARA A ANÁLISE DE INTERFERÊNCIAS}

Esta seção apresenta o enfoque probabilístico proposto neste artigo, que trata as razões portadora-interferência como variáveis aleatórias. O procedimento para a determinação das funções densidade de probabilidade dessas variáveis aleatórias é também descrito nesta seção. Com base neste enfoque probabilístico são definidas, na Subseção 3.1 figuras de mérito, representadas por métricas que permitem avaliar a necessidade e a dificuldade de coordenação entre pares de sistemas. A partir destas métricas, são definidas na Subseção 3.2 métricas adicionais voltadas para a avaliação da necessidade e dificuldade de coordenação quando múltiplos sistemas são considerados.

Observe que dados os sistemas interferente e interferido, ou seja, dados $j$ e $i$, o valor da razão portadora/interferênciatotal $(\mathcal{C} / \mathcal{I})_{i, k}^{j, \ell}$ depende das características do enlace interferente $\ell$ e das características do enlace interferido $k$. Note ainda que a ocorrência da situação de interferência associada ao valor $(\mathcal{C} / \mathcal{I})_{i, k}^{j, \ell}$ de razão portadora/interferência-total pressupõe a ocorrência dos seguintes eventos: (i) o enlace $\ell$ está sendo efetivamente utilizado pelo sistema interferente $j$ e; (ii) o enlace $k$ está sendo utilizado pelo sistema interferido $i$. No enfoque probabilístico, a cada um desses eventos é associ- 
ada uma probabilidade de ocorrência. Assim, a razão portadora/interferência-total $(\mathcal{C} / \mathcal{I})_{i}^{j}$ associada ao par $(j, i)$ de sistemas interferente e interferido é uma variável aleatória que depende de $\ell$, e $k$. Em conseqüência, a margem $x_{j i}$ de razão portadora/interferência-total, definida por

$$
x_{j i}(\ell, k)=\left(\frac{\mathcal{C}}{\mathcal{I}}\right)_{i, k_{[d B]}}^{j, \ell}-\eta_{i k_{[d B]}}
$$

onde $\eta_{i k_{[d B]}}$ denota o valor de razão portadora/interferênciatotal correspondente à máxima interferência aceitável sobre o enlace $k$ do sistema $i$, é também uma variável aleatória. A função densidade de probabilidade da margem $x_{j i}$ se escreve

$$
p_{x_{j i}}(X)=\sum_{K \in \mathcal{E}_{i}} p_{x_{j i} \mid k=K}(X) P(k=K)
$$

onde $p_{x_{j i} \mid k=K}(X)$ representa a função densidade de probabilidade da margem $x_{j i}$ dado que o enlace interferido do sistema $i$ é o enlace $K$.

Supondo-se que a probabilidade de um sistema qualquer (interferente ou interferido) utilizar qualquer um de seus possíveis enlaces é a mesma, tem-se

$$
P(k=K)=\frac{1}{\left\|\mathcal{E}_{i}\right\|} \quad ; K \in \mathcal{E}_{i}
$$

$$
P(\ell=L)=\frac{1}{\left\|\mathcal{E}_{j}\right\|} \quad ; L \in \mathcal{E}_{j}
$$

onde $\left\|\mathcal{E}_{i}\right\|$ e $\left\|\mathcal{E}_{j}\right\|$ denotam respectivamente o número de elementos de $\mathcal{E}_{i}$ e $\mathcal{E}_{j}$. Considerando-se (12), (11) se escreve

$$
p_{x_{j i}}(X)=\frac{1}{\left\|\mathcal{E}_{i}\right\|} \sum_{K \in \mathcal{E}_{i}} p_{x_{j i} \mid k=K}(X)
$$

Em (14), aA função densidade de probabilidade condicional $p_{x_{j i} \mid k=K}(X)$, da variável aleatória $x_{j i}$ dado o enlace interferido $(k=K)$, considera que a estação terrena vítima está localizada sempre no ponto mais desfavorável da área de cobertura correspondente ao feixe de transmissão do enlace $k$ que, conforme indicado na Seção 4.1.2, depende também do enlace interferente considerado $(\ell)$. Assim, $p_{x_{j i} \mid k=K}(X)$ em (14) é função apenas do enlace interferente $\ell=L$. Considerando-se que a probabilidade do sistema interferente utilizar qualquer um de seus enlaces é a mesma, ou seja, que (13) é válida, $p_{x_{j i} \mid k=K}(X)$ pode ser estimada pelo histograma normalizado (frequiência relativa de ocorrência) dos valores de $x_{j i}$ considerando-se todos os possíveis enlaces $\ell \in \mathcal{E}_{j}$, ou seja,

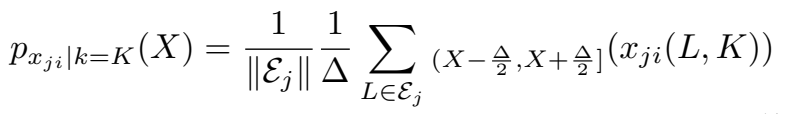

onde ${ }_{[a, b]}(\alpha)$ denota a função indicadora do intervalo $(a, b]$, definida por

$$
{ }_{(a, b]}(\alpha)=\left\{\begin{array}{lll}
1 & ; & \alpha \in(a, b] \\
0 & ; & \alpha \notin(a, b]
\end{array}\right.
$$

e $\Delta$ representa o tamanho do intervalo de quantização dos valores de $x_{j i}$ (“bin”).

\section{Consideração dos diferentes níveis de potência de trans- missão de uma mesma portadora}

Considerando que usualmente os valores das densidades de potência de transmissão das diversas emissões presentes no banco de dados da UIT são caracterizados através de intervalos (densidade de potência mínima e densidade de potência máxima), no enfoque probabilístico estas densidades de potência foram modeladas como variáveis aleatórias com distribuição de probabilidade uniforme nestes intervalos. Em particular, foram considerados três possíveis valores para cada uma destas variáveis aleatórias, todos com igual probabilidade. Os valores utilizados correspondem aos valores mínimo, máximo e médio (média feita em $\mathrm{W} / \mathrm{Hz}$ ) dessas densidades de potência. Assim, dado um par de sistemas (interferente $j$ e interferido $i$ ) e fixado um par de enlaces (interferente $\ell$ e interferido $k$ ), os três valores possíveis para a densidade de potência transmitida pela estação terrena interferente e os três valores possíveis para a densidade de potência transmitida pela estação terrena do sistema interferido originam nove valores equiprováveis para a razão portadora interferência no lance de subida (up-link). De maneira análoga, os três valores possíveis para a densidade de potência transmitida pelo satélite interferente e os três valores possíveis para a densidade de potência transmitida pelo satélite do sistema interferido originam também nove valores equiprováveis para a razão portadora interferência no lance de descida (downlink).

No cálculo da razão portadora/interferência-total, cada um dos nove valores de razão portadora-interferência de up-link é combinado com um dos nove valores de razão portadora-interferência de down-link dando origem a nove valores equiprováveis da razão portadora/interferência-total $(\mathcal{C} / \mathcal{I})_{i, k}^{j, \ell}$. Nesta combinação considerou-se que, para qualquer enlace, os valores mínimo, máximo e médio da densidade de potência de transmissão da estação terrena são respectivamente associados aos valores mínimo, máximo e médio da densidade de potência de transmissão do satélite utilizado pelo enlace. Os nove valores obtidos para $(\mathcal{C} / \mathcal{I})_{i, k}^{j, \ell}$ dão origem a nove valores equiprováveis para a margem $x_{j i}$ definida em (10). Assim, fixado o enlace $k$ do sistema interferido $i$, cada enlace $\ell$ do sistema interferente $j$ contribui com nove entradas para a formação do histograma normalizado que estima a função densidade de probabilidade condicional $p_{x_{j i} \mid k=K}(X)$ que aparece em (14).

\section{Exemplo}

As figuras 3 e 4 ilustram respectivamente as funções densidade de probabilidade $p_{x_{j i}}(X)$ e distribuição de probabilidade $F_{x_{j i}}(X)$ da margem $x_{j i}$, correspondentes à interferencia co-canal provocada por enlaces de um dado sistema da base de dados da UTI, aqui denominado USASAT-24Y (sistema $j$ ), com satélite localizado em uma dada posição orbital $\Phi^{\circ}$, em enlaces de um outro sistema desta mesma base de dados, aqui denominado B-SAT I (sistema $i$ ), com satélite na posição orbital $(\Phi-4)^{\circ}$. Na obtenção das curvas das figuras 3 e 4, foram considerados todos os possíveis enlaces de cada um dos sistemas envolvidos (200 no sistema USASAT$24 \mathrm{Y}$ e 16.082 no sistema B-SAT I). No levantamento dos his- 
togramas correspondentes a $p_{x_{j i} \mid k=K}(X)$, foram utilizados intervalos de quantização ("bins") de $0,2 \mathrm{~dB}$. No cálculo das margens $x_{j i}$, foi considerado um valor do limiar $\eta_{i k_{[d B]}}$ dado por

$$
\eta_{i k_{[d B]}}=\left(\frac{C}{N}\right)_{i, k_{[d B]}}^{r e q}+12,2
$$

onde $(C / N)_{i, k_{[d B]}^{r e q}}^{r}$ corresponde ao valor mínimo de razão portadora-ruído requerido para operação adequada do enlace $k$ do sistema $i$.

O mesmo procedimento foi utilizado na determinação das funções densidade de probabilidade $p_{x_{i j}}(X)$ e distribuição de probabilidade $F_{x_{i j}}(X)$ da margem $x_{i j}$, correspondentes à interferencia co-canal provocada por enlaces do sistema BSAT I em enlaces do sistema USASAT-24Y, apresentadas nas figuras 5 e 6.

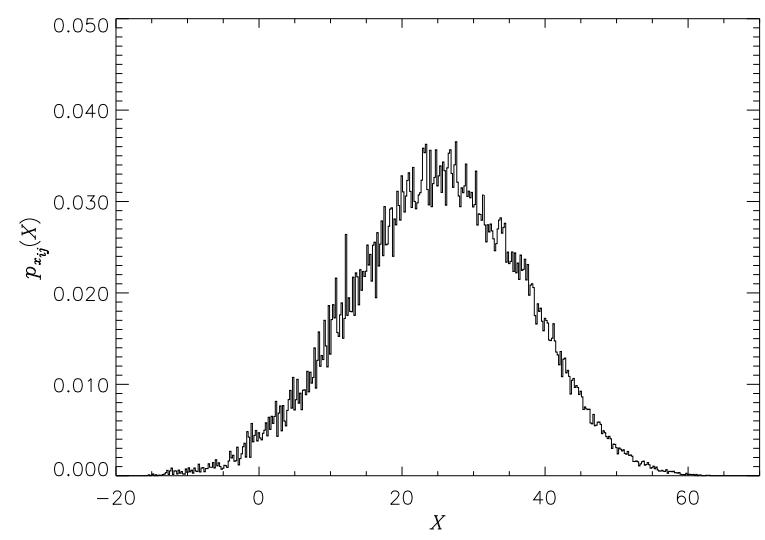

Figura 3. Função densidade de probabilidade da margem $x_{j i}$ correspondente a interferência co-canal de enlaces do sistema USASAT-24Y em enlaces do sistema B-SAT I

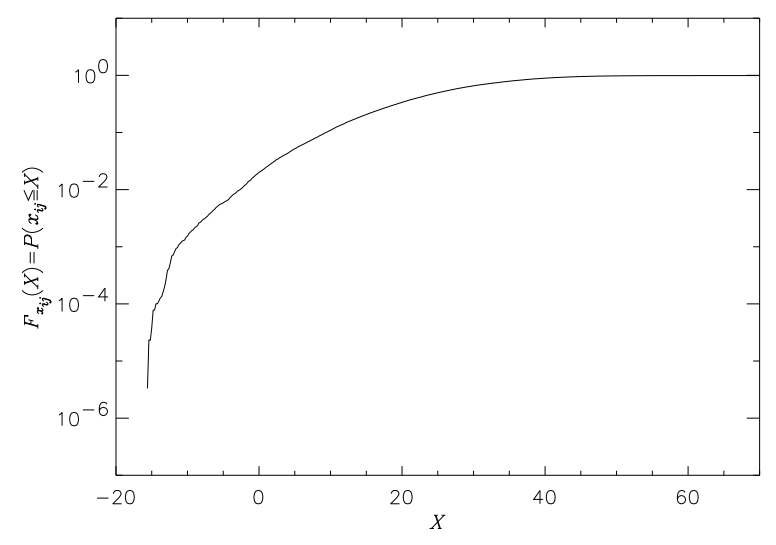

Figura 4. Função distribuição de probabilidade da margem $x_{j i}$ correspondente a interferência co-canal de enlaces do sistema USASAT-24Y em enlaces do sistema B-SAT I

\subsection{FIGURAS DE MÉRITO}

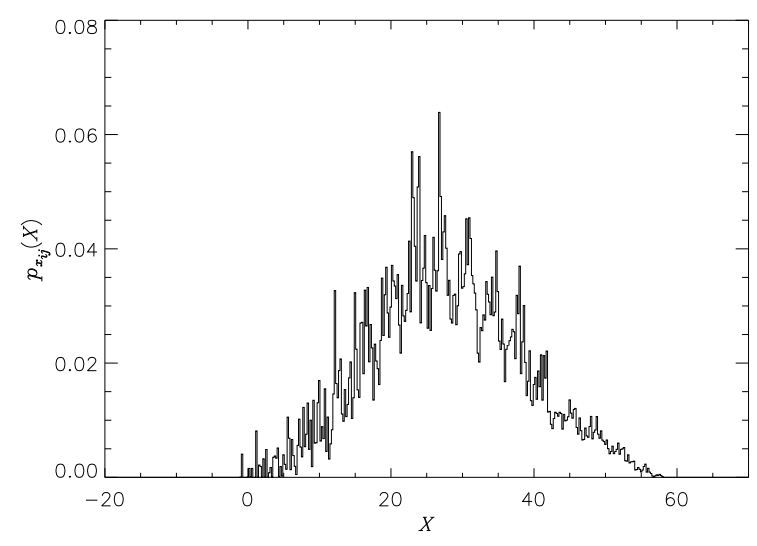

Figura 5. Função densidade de probabilidade da margem $x_{i j}$ correspondente a interferência co-canal de enlaces do sistema B-SAT I em enlaces do sistema USASAT-24Y

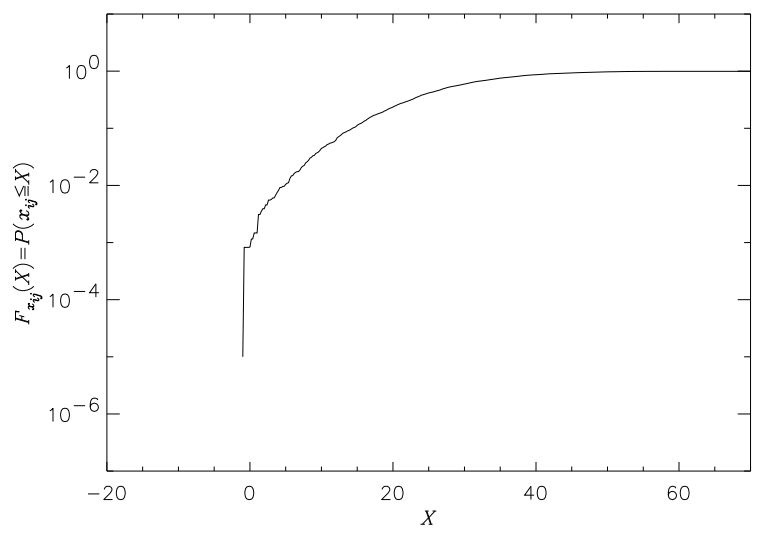

Figura 6. Função distribuição de probabilidade da margem $x_{i j}$ correspondente a interferência co-canal de enlaces do sistema B-SAT I em enlaces do sistema USASAT-24Y

Com base no enfoque probabilístico apresentado na Subseção 3, esta subseção introduz algumas figuras de mérito (métricas) voltadas para a análise do grau de utilização da órbita de satélites geoestacionários. Estas métricas permitem avaliar a necessidade e a dificuldade de coordenação entre sistemas de comunicação por satélite e suas definições são apresentadas a seguir.

\section{- uma medida da necessidade de coordenação}

Considerando que na avaliação da interferência entre dois sistemas, a ocorrência de margens negativas da razão portadora/interferência-total implica na coordenação entre os sistemas envolvidos, uma medida da necessidade de coordenação é dada pela probabilidade de ocorrência de margem negativa de razão portadora/interferência-tot. Assim, ao se avaliar a interferência de um dado sistema $j$ sobre um outro sistema $i$, a probabilidade de coordenação devido à interferência do sistema $j$ no sistema $i$ é dada por

$$
P_{(j \rightarrow i)}=P\left(x_{j i} \leq 0\right)=\int_{-\infty}^{0} p_{x_{j i}}(X) d X
$$


Considerando-se, por exemplo, a interferência do sistema USASAT-24Y no sistema B-SAT I, a probabilidade de coordenação entre os dois sistemas, devido à interferência do sistema USASAT-24Y no sistema B-SAT I, obtida da Figura 4, é relativamente pequena, sendo igual a $P_{(j \rightarrow i)}=F_{x_{j i}}(0)=2,00 \times 10^{-2}$. Observase ainda que a probabilidade de coordenação entre os dois sistemas, devido à interferência do sistema B-SAT I no sistema USASAT-24Y, obtida da Figura 6, é também bastante pequena, sendo igual a $P_{(i \rightarrow j)}=F_{x_{i j}}(0)=$ $8,24 \times 10^{-4}$.

\section{- uma medida da dificuldade de coordenação}

Havendo a necessidade de coordenação entre dois sistemas, é razoável supor que quanto maior for a incidência de valores elevados (em módulo) de margens negativas de razão portadora/interferência-total maiores serão as dificuldades para a solução das incompatibilidades. Assim, uma medida adequada da dificuldade de coordenação é dada pelo valor $r m s$ das margens negativas, dado por

$$
\begin{aligned}
\chi_{(j \rightarrow i)} & =\sqrt{E\left[x_{j i}^{2} \mid x_{j i} \leq 0\right]} \\
& =\left(\frac{1}{P_{(j \rightarrow i)}} \int_{-\infty}^{0} X^{2} p_{x_{j i}}(X) d X\right)^{\frac{1}{2}}
\end{aligned}
$$

Considerando-se, por exemplo, a interferência do sistema USASAT-24Y no sistema B-SAT I, o valor $r m s$ das margens negativas, devido à interferência do sistema USASAT-24Y no sistema B-SAT I, calculado utilizando-se (19) e a função densidade de probabilidade da Figura 3, é igual a $\chi_{(j \rightarrow i)}=5,09 \mathrm{~dB}$. Por outro lado, o valor $r m s$ das margens negativas devido à interferência do sistema B-SAT I no sistema USASAT-24Y, obtida utilizando-se (19) e a função densidade de probabilidade da Figura 5, é bastante inferior, sendo igual a $\chi_{(i \rightarrow j)}=0,79 \mathrm{~dB}$.

\section{- uma medida conjunta da necessidade e da dificul- dade de coordenação}

Podem existir situações em que as interferências produzidas por diferentes sistemas interferentes em um dado sistema vítima resultem em valores iguais de probabilidade de coordenação, porém com diferentes valores $r m s$ de margens negativas (diferentes dificuldades de coordenação). Por outro lado, podem existir também situações onde diferentes sistemas interferentes resultem na mesma dificuldade de coordenação (mesmo valor de $\chi$ ), porém com diferentes probabilidades de coordenação (necessidade de coordenação). Assim, as duas medidas definidas nos ítens anteriores devem ser levadas em consideração na avaliação do potencial interferente envolvendo um par de sistemas. Uma medida adequada do potencial interferente de um dado sistema $j$ em um dado sistema $i$, que leva em conta tanto a necessidade de coordenação quanto a dificuldade de coordenação, é dada pelo, aqui denominado "nível de coordenação devido à interferência de $j$ em $i$ ", definido por

$$
\mu_{(j \rightarrow i)}=\chi_{(j \rightarrow i)}^{2} P_{(j \rightarrow i)}=\int_{-\infty}^{0} X^{2} p_{x_{j i}}(X) d X
$$

Note que a métrica em (20) é sempre não negativa e que se ela é igual a zero não existem margens negativas devido à interferência de $j$ em $i$, ou seja, $P_{(j \rightarrow i)}=0$.

O nível de coordenação associado ao par formado pelos sistemas $j$ e $i$ é aqui definido por

$$
\mu_{(j, i)}=\mu_{(i, j)}=\mu_{(j \rightarrow i)}+\mu_{(i \rightarrow j)}
$$

Quanto mais baixo for o nível de coordenação $\mu_{(j, i)}$ entre dois sistemas, mais fácil a coordenação entre eles. Note que como a métrica em (20) é sempre não negativa, quando $\mu_{(j, i)}=0$ não há a necessidade de coordenação entre os sistemas, já que cada uma das parcelas em (21) é nula.

Considerando-se, por exemplo, as interferências entre os sistemas USASAT-24Y $(j)$ e B-SAT I $(i)$ tem-se, com base nas funções densidade de probabilidade das figuras 3 e 5 , que $\mu_{(j \rightarrow i)}=5,18 \times 10^{-1}, \mu_{(i \rightarrow j)}=5,00 \times 10^{-4}$ e $\mu_{(j, i)}=\mu_{(i, j)}=5,185 \times 10^{-1}$, que são valores relativamente pequenos para estas quantidades, significando que a coordenação entre os sistemas USASAT-24Y e BSAT I não deve ser difícil.

\subsection{CONSIDERAÇÃO DE MÚLTIPLOS SIS- TEMAS INTERFERENTES}

Quando os recursos da órbita de satélites geoestacionários e do espectro de frequiências são compartilhados por vários sistemas, é usual, para fins de análise de desempenho dos sistemas, se considerar o efeito agregado das diversas parcelas de interferência sobre o sistema interferido. Entretanto, para fins de coordenação entre os sistemas, como as negociações (coordenações) ocorrem de forma bilateral, as informações sobre os niveis de interferência entre pares de sistemas tornam-se mais relevantes para o processo de coordenação do que as informações sobre os níveis de interferência agregada. Assim, na avaliação de um ambiente interferente que contenha múltiplos sistemas, é adequada a utilização de uma figura de mérito que dependa das métricas associadas a cada possível par de sistemas.

Com o objetivo de definir uma métrica adequada, considere um conjunto $\mathcal{S}$ de sistemas existentes e seja $i$ um sistema novo que deverá compartilhar os recursos da órbita de satélites geoestacionários e do espectro de frequiências com os sistemas existentes. Uma métrica que reflete a necessidade/dificuldade de coordenação entre o sistema $i$ e os sistemas existentes, devido à interferência dos sistema existentes sobre o sistema $i$ é dada pelo "nível de coordenação devido à interferência dos sistemas existentes no sistema i”, definido por

$$
\mu_{(\mathcal{S} \rightarrow i)}=\sum_{j \in \mathcal{S}} \mu_{(j \rightarrow i)}
$$


onde as parcelas do somatório são obtidas por meio de (20).

De modo análogo, uma métrica que reflete a necessidade/dificuldade de coordenação entre o sistema $i$ e os sistemas existentes, devido à interferência do sistema $i$ sobre os sistemas existentes é dada pelo "nível de coordenação devido à interferência do sistema i nos sistemas existentes", definido por

$$
\mu_{(i \rightarrow \mathcal{S})}=\sum_{j \in \mathcal{S}} \mu_{(i \rightarrow j)}
$$

A partir das métricas em (22) e (23), define-se o "nível de coordenação entre o sistema i e os sistemas existentes" como

$$
\mu_{(\mathcal{S}, i)}=\mu_{(i, \mathcal{S})}=\mu_{(\mathcal{S} \rightarrow i)}+\mu_{(i \rightarrow \mathcal{S})}
$$

A métrica em (24) reflete a necessidade/dificuldade de coordenação entre o novo sistema $i$ e os sistemas existentes. Quanto mais baixo o nível de coordenação $\mu_{(\mathcal{S}, i)}$, mais fácil a coordenação entre o sistema $i$ e os sistemas existentes. Quando $\mu_{(\mathcal{S}, i)}=0$ não há necessidade de coordenação do sistema $i$ com qualquer dos sistemas em $\mathcal{S}$ (sistemas existentes).

A dificuldade de coordenação associada a uma dada posição orbital $\phi$ será então caracterizada pelo nível de coordenação entre os sistemas existentes e um hipotético sistema padrão colocado na posição orbital $\phi$, denotado por $\mu_{(\mathcal{S}, \phi)}$, e calculado através de $(24)$.

Caracterizações parciais da dificuldade de coordenação associada à posição orbital $\phi$ são dadas pela dificuldade de coordenação devido à interferência dos sistemas existentes no sistema padrão e pela dificuldade de coordenação devida à interferência do sistema padrão nos sistemas existentes. Estas caracterizações parciais são respectivamente calculadas através de (22) e (23) e denotadas por $\mu_{(\mathcal{S} \rightarrow \phi)}$ e $\mu_{(\phi \rightarrow \mathcal{S})}$.

\section{EXEMPLO DE APLICAÇ̃̃O DO EN- FOQUE PROBABILÍSTICO}

Exemplos da utilização das métricas propostas neste trabalho na elaboração de diagnósticos de posições orbitais considerando o ambiente interferente produzido por sistemas de comunicações que compartilham a OSG são apresentados nesta seção. Os dados utilizados nesses exemplos foram extraídos da Base de Dados Space Radiocommunications Stations da ITU (Edição de setembro de 2001) [6].

\subsection{ALGUNS ASPECTOS DO PROCEDI- MENTO DE CÁLCULO}

Esta seção destaca alguns aspectos dos procedimentos de cálculo levados a efeito na implementação da metodologia descrita na Seção 2. A Seção 4.1.1 aborda os pré-processamentos efetuados sobre os dados disponíveis no banco de dados da UIT enquanto a Seção 4.1.2 tece considerações sobre o posicionamento geográfico das estações terrenas envolvidas nos cálculos de interferência.

\subsubsection{PRÉ-PROCESSAMENTO DOS DADOS}

Dado o grande volume de dados a serem considerados na elaboração de um diagnóstico da utilização do arco orbital baseado na metodologia descrita na Seção 2, é importante que a implementação da metodologia proposta seja feita de maneira eficiente de modo a reduzir o tempo de computação requerido. Com este objetivo, os processamentos de dados que não dependem da situação de interferência em análise foram efetuados previamente e seus resultados armazenados em banco de dados. Estes pré-processamentos são descritos a seguir.

\section{Identificação dos enlaces possíveis}

Para cada sistema de comunicação por satélite, foi identificado o conjunto dos enlaces que poderiam ser implementados no sistema. Na identificação desses enlaces foram considerados os feixes de origem e destino (recepção e transmissão) com os respectivos diagramas de radiação, a potência de transmissão, as antenas das estações terrenas e seus diagramas de radiação, o tipo de emissão (portadora) e sua freqüência. A obtenção deste conjunto de enlaces foi feita através de um pré-processamento dos dados e levou em conta a informação sobre a conectividade entre os transponders do sistema transponder strapping. Assim, a cada um dos sistemas considerados foi associado um conjunto de possíveis enlaces.

No procedimento aqui adotado considerou-se que se um dado tipo de emissão $E$ está presente no conjunto de emissões que podem ser recebidas pelo feixe de recepção $R$ e também no conjunto de emissões que podem ser transmitidas pelo feixe de transmissão $T$, então, contanto que os feixes $R$ e $T$ possam ser conectados (transponder strapping), existiria, em princípio, um enlace possível envolvendo a emissão $E$, com lance de subida utilizando o feixe $E$ e lance de descida utilizando o feixe $T$. Entretanto, para garantir a viabilidade deste enlace, considerou-se o requisito técnico de que a razão portadora-ruído térmico $(C / N)$ calculada deve ser superior ao valor de $(C / N)$ especificado como requerido para o enlace. Enlaces para os quais esta condição não foi satisfeita foram excluídos do conjunto de enlaces possíveis.

\section{Classificação dos enlaces}

A quantidade dos enlaces associados a cada sistema, gerados conforme descrito no item anterior, pode ser bastante grande (alguns sistemas possuem um conjunto de enlaces possíveis contendo centenas de milhares de enlaces). Na verdade, verifica-se que muitos desses enlaces possuem o mesmo potencial interferente e a mesma sensibilidade à interferência co-canal e podem portanto ser agrupados em uma mesma classe. Este é o caso, por exemplo, de enlaces que só se diferenciam pela freqüência da emissão. Assim, para cálculo de interferência, todos os enlaces em uma mesma classe podem ser representados por um único enlace com multiplicidade igual ao número de enlaces na classe. O número de enlaces a serem considerados nos cálculos de interferência fica reduzido ao número de classes. Em situações nas quais 
o número de classes de enlaces equivalentes seja ainda muito grande, é possível particionar o conjunto de enlaces possíveis em classes de enlaces quasi-equivalentes, definidas como sub-conjuntos de enlaces que têm tanto potenciais interferentes quanto sensibilidades à interferência comparáveis. Assim, como no caso de classes de enlaces equivalentes, para cálculo de interferência, todos os enlaces em uma mesma classe de enlaces quasi-equivalentes são representados por um único enlace, adequadamente escolhido na classe, com multiplicidade igual ao número de enlaces na classe. A obtenção das classes de enlaces quasi-equivalentes pode ser feita através de técnicas de quantização vetorial. O procedimento utilizado na obtenção dessas classes é descrito no Apêndice.

\section{Geração de pontos nas fronteiras das áreas de cobertura}

Como o procedimento para a identificação das posições mais desfavoráveis para a localização das estações transmissorainterferente e receptora-interferida envolve uma busca ao longo das fronteiras das áreas de serviço, foram gerados e armazenados, para cada um dos feixes (transmissão e recepção) de cada uma das redes de comunicação por satélite consideradas, pontos de teste localizados sobre estas fronteiras. As áreas de serviço foram definidas como a região sobre a superfície da Terra correspondente à interseção da área interna ao contorno de $-3 \mathrm{~dB}$ do feixe com a àrea de visibilidade do satélite. A obtenção do conjunto de pontos de teste associado a cada feixe (à área de serviço correspondente ao feixe) foi feita através de um pré-processamento dos dados e utilizou algoritmos especialmente desenvolvidos para este fim. Estes algoritmos utilizam o método de triangulação de Delaunay [7] para realizar interpolações bidimensionais nos diagramas de radiação das antenas dos satélites. A Figura 7 ilustra a disposição dos pontos de teste obtidos para um dos feixe de transmissão do sistema B-SAT I.

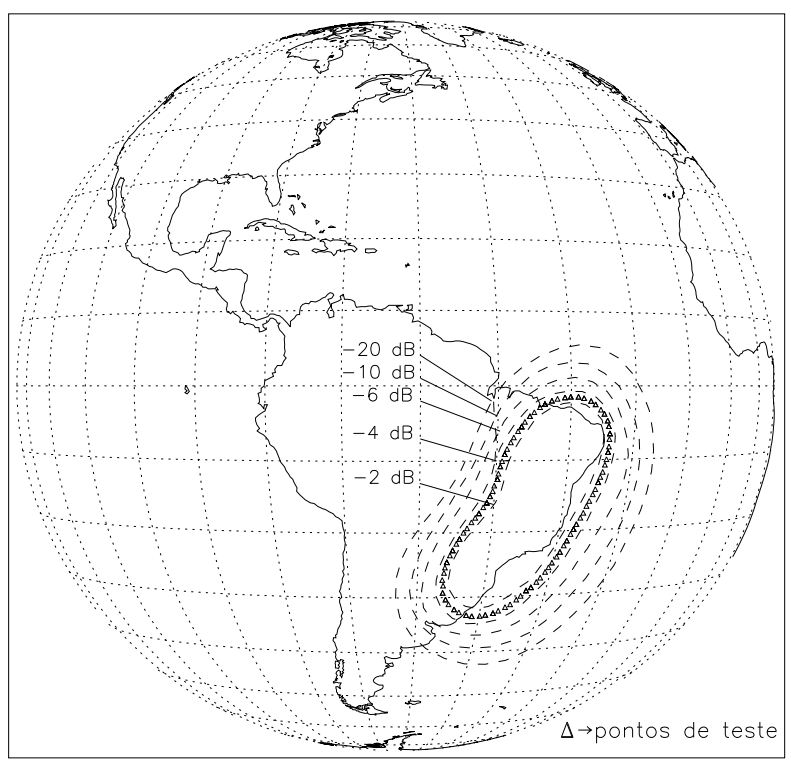

Figura 7. Pontos de teste obtidos para um dos feixes de transmissão do sistema B-SAT I (sobre o contorno de $-3 \mathrm{~dB}$ )

\subsubsection{POSICIONAMENTO DAS ESTAÇÕES TRANSMISSORAS E RECEPTORAS}

Conforme ilustrado na Figura 8, sejam $A_{g_{2}^{\prime}}$ e $A_{g_{3}^{\prime}}$ as áreas de serviço associadas respectivamente aos feixes de recepção e transmissão do satélite do sistema interferente. De modo análogo, sejam $A_{g_{2}}$ e $A_{g_{3}}$ as áreas de serviço associadas respectivamente aos feixes de recepção e transmissão do satélite do sistema interferido.

\section{- posicionamento da estação terrena transmissora do} sistema interferente

Conforme mencionado na Seção 2.1 supõe-se que a estação terrena transmissora interferente está localizada na posição geográfica mais desfavorável de sua área de serviço $A_{g_{2}^{\prime}}$, ou seja, na posição que correspondente ao maior nível de interferência de up-link. Assim, esta posição é definida pelo ponto de $A_{g_{2}^{\prime}}$ que apresenta o maior ganho do feixe de recepção do satélite interferido (maior valor de $g_{2}$ ). Note que caso o ponto da superifície da Terra que corresponde ao máximo valor de $g_{2}$ pertença a $A_{g_{2}^{\prime}}$ (o que pode ocorrer quando as áreas de serviço $A_{g_{2}^{\prime}}$ e $A_{g_{2}}$ não são disjuntas) a estação terrena transmissora do sistema interferente deverá ser colocada neste ponto. Caso contrário, o ponto de $A_{g_{2}^{\prime}}$ correspondente ao maior valor de $g_{2}$ pertence à fronteira de $A_{g_{2}^{\prime}} \mathrm{e}$ é obtido através de uma busca sobre o conjunto de pontos desta fronteira.

\section{- posicionamento da estação terrena receptora do sis- tema interferido}

Conforme mencionado na Seção 2.1 supõe-se que a estação terrena receptora interferida está localizada na posição geográfica mais desfavorável de sua área de serviço $A_{g_{3}}$, ou seja, na posição que correspondente ao maior nível de interferência de down-link. Assim, esta posição é definida pelo ponto de $A_{g_{3}}$ que apresenta o maior ganho do feixe de transmissão do satélite interferente (maior valor de $g_{3}^{\prime}$ ). Note que caso o ponto da superifície da Terra que corresponde ao máximo valor de $g_{3}^{\prime}$ pertença a $A_{g_{3}}$ (o que pode ocorrer quando as áreas de serviço $A_{g_{3}}$ e $A_{g_{3}^{\prime}}$ não são disjuntas) a estação terrena receptora do sistema interferido deverá ser colocada neste ponto. Caso contrário, o ponto de $A_{g_{3}}$ correspondente ao maior valor de $g_{3}^{\prime}$ pertence à fronteira de $A_{g_{3}}$ e é obtido através de uma busca sobre o conjunto de pontos desta fronteira gerado de acordo com o descrito na Subseção 4.1.1.

- posicionamento da estação terrena transmissora do sistema interferido

Supõe-se que a estação terrena transmissora do sistema interferido está localizada na posição mais desfavorável da área de serviço $A_{g_{2}}$, ou seja, na posição que corresponde ao menor nível de sinal de up-link. Assim, esta posição é definida pelo ponto da fronteira de $A_{g_{2}}$ que tem a maior distância ao satélite do sistema interferido (maior percurso no lance de subida). 


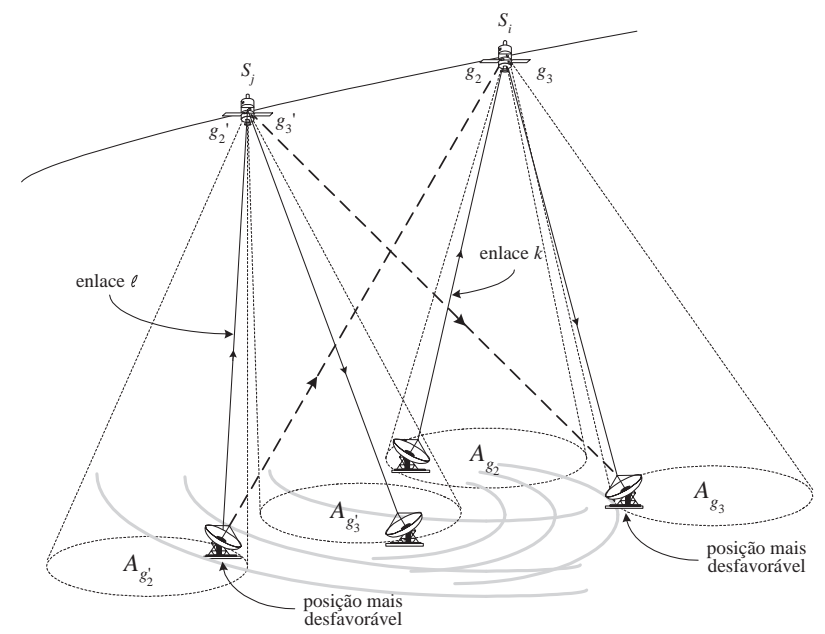

Figura 8. Posicionamento das estações terrenas transmissoras e receptoras (ilustração do caso em que não há superposição das áreas de serviço). $S_{j}$ é o satélite do sistema interferente e $S_{i}$ o satélite do sistema interferido.

\subsection{DIAGNÓSTICO DE UMA POSIÇÃO OR- BITAL}

Nesta seção as figuras de mérito (métricas) introduzidas na Seção 2, que refletem a necessidade e dificuldade de coordenação entre sistemas de comunicação por satélite, foram utilizadas para produzir um diagnóstico detalhado de uma dada posição orbital no que diz respeito ao ambiente interferente produzido por sistemas que compartilham a Banda $\mathrm{Ku}(14 / 10-12 \mathrm{GHz})$. Em particular foi avaliada uma posição orbital $\Phi$, considerando-se o conjunto de sistemas que operam satélites localizados na vizinhança orbital $\left[(\Phi-9)^{\circ},(\Phi+9)^{\circ}\right]$, conforme indicado na Tabela 1. Foram calculadas os valores relacionados à necessidade $\left(P_{(j \rightarrow i)}\right)$, dificuldade $\left(\chi_{(j \rightarrow i)}\right)$ e níveis de coordenação $\left(\mu_{(j \rightarrow i)}\right)$ envolvendo um hipotético sistema padrão colocado na posição orbital em exame e cada um dos sistemas relacionados na Tabela 1. O sistema padrão adotado tem características idênticas às do sistema B-SAT I (que originam 16082 enlaces possíveis).

A Tabela 2 apresenta os valores relacionados à necessidade, dificuldade e nível de coordenação devidos à interferência do sistema padrão em cada um dos sistemas considerados quando o satélite do sistema padrão ocupa a posição orbital $\Phi^{\circ}$, enquanto que a Tabela 3 fornece os resultados obtidos para estas métricas considerando-se a interferência de cada um dos sistemas existentes no sistema padrão. Os resultados apresentados na Tabela 4 levam em consideração os dois sentidos de interferência, apresentando, para a posição orbital em análise, os valores de nível de coordenação associado a cada par sistema-padrão/sistema-existente considerado. A última linha da Tabela 4 mostra o valor do nível de coordenação associado à posição orbital em exame, e correspondente ao nível de coordenação entre o conjunto de sistemas considerados e o sistema padrão (obtido, por sua vez, somando-se os valores apresentados nas linhas desta tabela).
Tabela 1. Sistemas com emissões na Banda $K u$ e satélite no arco orbital $\left[(\Phi-9)^{\circ},(\Phi-9)^{\circ}\right]$

\begin{tabular}{||c|c|c||}
\hline \hline número & sistema & posição orbital \\
\hline \hline 1 & GEO-1 & $\Phi-9$ \\
2 & GEO-2 & $\Phi-9$ \\
3 & GEO-3 & $\Phi-7$ \\
4 & GEO-4 & $\Phi-6$ \\
5 & GEO-5 & $\Phi-5$ \\
6 & GEO-6 & $\Phi-4$ \\
7 & GEO-7 & $\Phi-4$ \\
8 & GEO-8 & $\Phi-2.5$ \\
9 & GEO-9 & $\Phi-2$ \\
10 & GEO-10 & $\Phi+2$ \\
11 & GEO-11 & $\Phi+2$ \\
12 & GEO-12 & $\Phi+4$ \\
13 & GEO-13 & $\Phi+4$ \\
14 & GEO-14 & $\Phi+5$ \\
15 & GEO-15 & $\Phi+6.8$ \\
16 & GEO-16 & $\Phi+7.5$ \\
\hline \hline
\end{tabular}

Um exame dos valores apresentados na Tabela 4 evidencia que os dois maiores níveis de coordenação bilateral envolvendo um sistema padrão operando na Banda $K u$ com satélite localizado na posição orbital $\Phi$ analisada estão associados às interferências entre este sistema e o sistema GEO8, seguido do sistema GEO-12. Ressalte-se ainda que, de acordo com os resultados apresentados nas tabelas 2 e 3, a maior contribuição para estes níveis de coordenação bilateral é devida, em ambos os casos, às interferências produzidas pelo sistema padrão. O terceiro, quarto e quinto maiores esforços de coordenação bilateral envolvem, respectivamente, o sistema GEO-10, GEO-16 e GEO-14, sendo devidos, em todos estes 3 últimos casos, em sua maior parte, às interferências produzidas por estes sistemas no sistema padrão.

\subsection{DIAGNÓSTICO DE ARCO ORBITAL}

A análise de um arco orbital qualquer pode ser feita repetindo-se o procedimento utilizado no exemplo da Seção 4.2 para cada uma das posições orbitais do arco. A título de exemplo considerou-se um arco orbital de $135^{\circ}$ definido por $\left[(\Phi-57)^{\circ},(\Phi+78)^{\circ}\right]$, onde $\Phi$ é a posição orbital abordada na seção anterior. Este arco orbital foi avaliado a passos de $0.5^{\circ}$ considerando-se um sistema padrão com as mesmas característica do sistema B-SAT I.

A Figura 9 apresenta a variação dos níveis de coordenação, $\mu(\mathcal{S}, \phi)$, devido à interferência entre o conjunto de sistemas constantes da base de dados da UIT [6] com emissões na Banda $K u$ e o sistema padrão, com a posição orbital $\phi$ $\left(\phi \in\left[(\Phi-57)^{\circ},(\Phi+78)^{\circ}\right]\right)$ do satélite do sistema padrão. Quanto menor o valor associado a uma dada posição orbital do arco em análise mais fácil a coordenação entre os sistemas existentes e um novo sistema com satélite colocado nesta posição. Assim, o resultado apresentado no gráfico da 
Tabela 2. Métricas associadas às interferências geradas pelo sistema padrão quando colocado na posição orbital $\Phi^{\circ}$

\begin{tabular}{|c|c|c|c|}
\hline interferente $(j) \rightarrow$ interferido $(i)$ & $P_{(j \rightarrow i)}$ & $\chi_{(j \rightarrow i)}[\mathrm{dB}]$ & $\mu_{(j \rightarrow i)}\left[\mathrm{dB}^{2}\right]$ \\
\hline PADRÃO $\rightarrow$ GEO-1 & 0.38748780 & 7.4940 & 21.76140022 \\
\hline PADRÃO $\rightarrow$ GEO-2 & 0.00358662 & 1.8224 & 0.01191218 \\
\hline PADRÃO $\rightarrow$ GEO-3 & 0.06045516 & 4.3898 & 1.16497266 \\
\hline PADRÃO $\rightarrow$ GEO-4 & 0.02684244 & 3.6603 & 0.35963780 \\
\hline PADRÃO $\rightarrow$ GEO-5 & 0.11344650 & 5.7749 & 3.78335905 \\
\hline PADRÃO $\rightarrow$ GEO-6 & 0.19607021 & 6.7707 & 8.98834229 \\
\hline PADRÃO $\rightarrow$ GEO-7 & 0.00082411 & 0.7943 & 0.00052000 \\
\hline PADRÃO $\rightarrow$ GEO-8 & 0.78519636 & 14.5217 & 165.58283997 \\
\hline PADRÃO $\rightarrow$ GEO-9 & 0.36740297 & 9.2755 & 31.60935593 \\
\hline $\mathrm{PADRÃO} \rightarrow$ GEO-10 & 0.41353247 & 10.4558 & 45.20866013 \\
\hline PADRÃO $\rightarrow$ GEO-11 & 0.36705709 & 9.2491 & 31.39995575 \\
\hline PADRÃO $\rightarrow$ GEO- 12 & 0.72067058 & 11.4192 & 93.97351837 \\
\hline $\mathrm{PADRÃO} \rightarrow$ GEO-13 & 0.15514530 & 6.6532 & 6.86743164 \\
\hline PADRÃO $\rightarrow$ GEO-14 & 0.27208570 & 9.0168 & 22.12136269 \\
\hline $\mathrm{PADRÃO} \rightarrow$ GEO-15 & 0.00000000 & 0.0000 & 0.00000000 \\
\hline $\mathrm{PADRÃO} \rightarrow$ GEO-16 & 0.15126385 & 12.7638 & 24.64290237 \\
\hline
\end{tabular}

Tabela 3. Métricas associadas às interferências sofridas pelo sistema padrão quando colocado na posição orbital $\Phi^{\circ}$

\begin{tabular}{|c|c|c|c|}
\hline interferente $(j) \rightarrow$ interferido $(i)$ & $P_{(j \rightarrow i)}$ & $\chi_{(j \rightarrow i)}[\mathrm{dB}]$ & $\mu_{(j \rightarrow i)}\left[\mathrm{dB}^{2}\right]$ \\
\hline GEO-1 $\rightarrow$ PADRÃO & 0.13559166 & 5.8794 & 4.68705273 \\
\hline GEO-2 $\rightarrow$ PADRÃO & 0.00515801 & 3.8313 & 0.07571302 \\
\hline GEO-3 $\rightarrow$ PADRÃO & 0.06268082 & 4.4437 & 1.23775077 \\
\hline GEO-4 $\rightarrow$ PADRÃO & 0.17756906 & 17.7233 & 55.77746201 \\
\hline GEO-5 $\rightarrow$ PADRÃO & 0.11532803 & 5.7913 & 3.86796808 \\
\hline GEO-6 $\rightarrow$ PADRÃO & 0.26797318 & 8.4102 & 18.95400810 \\
\hline GEO-7 $\rightarrow$ PADRÃO & 0.01999116 & 5.0925 & 0.51843888 \\
\hline GEO-8 $\rightarrow$ PADRÃO & 0.47839677 & 12.2104 & 71.32627106 \\
\hline GEO-9 $\rightarrow$ PADRÃO & 0.36803260 & 9.2967 & 31.80873299 \\
\hline GEO-10 $\rightarrow$ PADRÃO & 0.52167994 & 11.2683 & 66.23991394 \\
\hline GEO-11 $\rightarrow$ PADRÃO & 0.36975265 & 9.2883 & 31.89921379 \\
\hline GEO-12 $\rightarrow$ PADRÃO & 0.39282310 & 9.2105 & 33.32419968 \\
\hline GEO-13 $\rightarrow$ PADRÃO & 0.15982319 & 6.6933 & 7.16020441 \\
\hline GEO-14 $\rightarrow$ PADRÃO & 0.39178818 & 10.6918 & 44.78720093 \\
\hline GEO-15 $\rightarrow$ PADRÃO & 0.23429930 & 7.3773 & 12.75154495 \\
\hline GEO-16 $\rightarrow$ PADRÃO & 0.38203025 & 10.7348 & 44.02394485 \\
\hline \multicolumn{4}{|c|}{$\mu_{\left(\mathcal{S} \rightarrow \Phi^{\circ}\right)}=428.43963623$} \\
\hline
\end{tabular}

Figura 9 reflete também o grau de congestionamento associado a uma dada posição orbital do arco considerado, permitindo uma avaliação das posições orbitais mais favoráveis para serem ocupadas na Banda $K u$. Na Figura 9, o valor correspondente a $\phi=\Phi(\phi-\Phi=0)$ é igual à raiz quadrada do valor de $\mu_{\left(\Phi^{\circ}, \mathcal{S}\right)}$ obtido na Seção 4.2 para a posição orbital $\Phi$ (ver Tabela 4). Observa-se que ao longo do arco orbital considerado nesta figura existem posições orbitais um pouco mais favoráveis do que a posição $\phi=\Phi$. Este é o caso, por exemplo, das posições orbitais $\phi=\Phi-57, \phi=\Phi-41 \mathrm{e}$ $\phi=\Phi+16$, assinaladas na Figura 9. Assim, se por exemplo deseja-se escolher uma posição orbital numa vizinhança de $20^{\circ}$ em torno da posição orbital $\Phi$, a melhor escolha seria a posição orbital $\phi=\Phi+16$.

\section{CONCLUSÃO}

Este artigo propôs um enfoque probabilístico para a análise do ambiente interferente envolvendo satélites geoestacinários visando 'a elaboração de diagnósticos de arcos orbitais. Estes diagnósticos permitem a avaliação do potencial de cada posição orbital quanto à sua localização no arco orbital e às dificuldades de coordenação entre redes de comunicação por satélite (processo de negociação bilateral para solucionar eventuais problemas de interferência). A abordagem pro- 
Tabela 4. Métricas associadas às interferências entre os sistemas existentes e o sistema padrão quando colocado na posição orbital $\Phi^{\circ}$

\begin{tabular}{||l|c||}
\hline \hline par de sistemas $(i, j)$ & $\mu_{(j, i)}=\mu_{(i, j)}\left[\mathrm{dB}^{2}\right]$ \\
\hline \hline GEO-1, PADRÃO & 26.44845200 \\
GEO-2, PADRÃO & 0.08762521 \\
GEO-3, PADRÃO & 2.40272331 \\
GEO-4, PADRÃO & 56.13710022 \\
GEO-5, PADRÃO & 7.65132713 \\
GEO-6, PADRÃO & 27.94235039 \\
GEO-7, PADRÃO & 0.51895887 \\
GEO-8, PADRÃO & 236.90911865 \\
GEO-9, PADRÃO & 63.41809082 \\
GEO-10, PADRÃO & 111.44857788 \\
GEO-11, PADRÃO & 63.29917145 \\
GEO-12, PADRÃO & 127.29771423 \\
GEO-13, PADRÃO & 14.02763557 \\
GEO-14, PADR $\tilde{O} O$ & 66.90856171 \\
GEO-15, PADRÃO & 12.75154495 \\
GEO-16, PADRÃO & 68.66684723 \\
\hline \hline \multicolumn{2}{|c|}{$\mu_{\left(\mathcal{S}, \Phi^{\circ}\right)}=\mu_{\left(\Phi^{\circ}, \mathcal{S}\right)}=885.91583252$} \\
\hline \hline \multicolumn{2}{|c|}{}
\end{tabular}

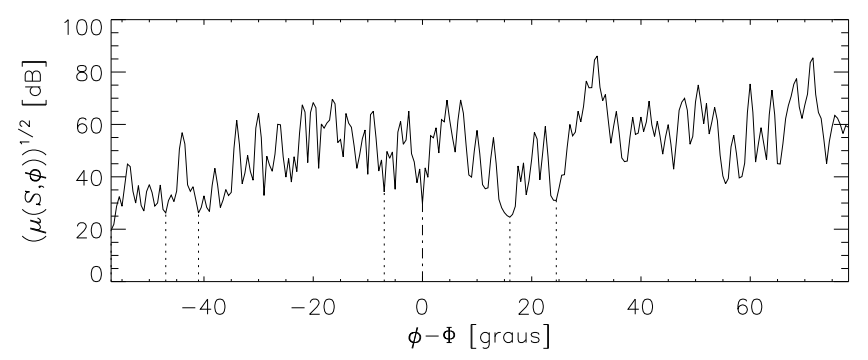

Figura 9. Nível de coordenação $\mu(\mathcal{S}, \phi)$ ao longo do arco orbital de interesse

babilística permite uma avaliação do congestionamento orbital com um esforço computacional muito inferior ao requerido por uma análise determinística detalhada, como aquela uitilizada pela UIT, sendo portanto mais adequada para a avaliação preliminar de um ambiente interferente, que apresenta constantes alterações, visando a escolha de posições orbitais para a localização de satélites geoestacionários de comunicações.

$\mathrm{O}$ enfoque proposto utiliza métricas que refletem tanto a necessidade quanto a dificuldade esperada no processo de coordenação entre redes de comunicações por satélite. Foram apresentados exemplos numéricos que ilustram a aplicação da metodologia proposta à situações práticas envolvendo redes de comunicações por satélite notificadas junto à UIT e cujas características técnicas estão disponibilizadas na Base de Dados Space Radiocommunications Stations da UIT. Esta nova metodologia foi utilizada recentemente num amplo estudo realizado para a Agência Nacional de Telecomunicações voltado para a geração de diagnósticos dos arcos orbitais de interesse do Brasil nas Bandas $\mathrm{C}, \mathrm{Ka}$ e $\mathrm{Ku}$.

\section{APÊNDICE}

\section{A. GERAÇÃO DE CLASSES DE EN- LACES EQUIVALENTES OU QUASI- EQUIVALENTES}

O agrupamento em classes foi feito para enlaces que possuem os mesmos feixes de recepção e transmissão do satélite, ou seja, enlaces que possuem os mesmos diagramas de radiação $g_{2}(\phi)$ e $g_{3}(\eta)$. Considerou-se que o diagrama de radiação $g_{1}(\theta)$ é função apenas do ganho $g_{1}(0)$ da antena e do ganho $e_{1}(\theta)$ dos lóbulos laterais, ou seja,

$$
g_{1}(\theta)=f_{1}\left(g_{1}(0), e_{1}(\theta)\right)
$$

De maneira análoga, considerou-se que

$$
g_{4}(\xi)=f_{4}\left(g_{4}(0), e_{4}(\xi)\right)
$$

Seja $\mathbf{x}$ o vetor do ${ }^{4}$ dado por

$$
\mathbf{x}=\left(\begin{array}{c}
p_{1} g_{1}(0) \\
p_{3} \\
g_{4}(0) \\
(C / N)_{r e q}
\end{array}\right)
$$

Considerando-se a definição de x e as expressões (4) a (8), conclui-se que enlaces que utilizem os mesmos feixes de recepção e transmissão do satélite e que tenham o mesmo conjunto de valores $\mathbf{x}$ e os mesmos padrões $e_{1}(\cdot)$ e $e_{4}(\cdot)$ para os ganhos de lóbulos laterais são equivalentes no que diz respeito ao cálculo de interferência. Isto significa que, mantendo-se a posição geográfica da estação terrena transmissora, tais enlaces causarão o mesmo nível de interferência em um dado enlace de um outro sistema e, mantendo-se a posição geográfica da estação terrena receptora, tais enlaces sofrerão o mesmo nível de interferência de um dado enlace de outro sistema. Assim o conjunto $\mathcal{E}$ de todos os enlaces que utilizam os mesmos feixes de recepção e transmissão do satélite pode ser particionado em classes de enlaces equivalentes (cada uma delas formada por enlaces que possuam os mesmos padrões $e_{1}(\cdot)$ e $e_{4}(\cdot)$ para os ganhos de lóbulos laterais e o mesmo valor de $\mathbf{x}$ ).

Em situações nas quais o número de classes de enlaces equivalentes seja ainda muito grande, é possível particionar o conjunto $\mathcal{E}$ de enlaces possíveis em classes de enlaces quasiequivalentes (cada uma delas formada por enlaces que possuam os mesmos padrões $e_{1}(\cdot)$ e $e_{4}(\cdot)$ para os ganhos de lóbulos laterais e valores de $\mathbf{x}$ próximos). Isto significa que, mantendo-se a posição geográfica da estação terrena transmissora, enlaces quasi-equivalentes causarão níveis de interferência comparáveis em um dado enlace de um outro sistema e, mantendo-se a posição geográfica da estação terrena receptora, tais enlaces sofrerão níveis de interferência comparáveis de um dado enlace de outro sistema. Assim, como no caso de classes de enlaces equivalentes, para cálculo de interferência, todos os enlaces em uma mesma classe de enlaces quasi-equivalentes são representados por um único enlace, adequadamente escolhido na classe, com multiplicidade igual ao número de enlaces na classe. A obtenção das classes de 
enlaces quasi-equivalentes pode ser feita através de técnicas de quantização vetorial, como o algoritmo LBG descrito em [5], aplicada ao conjunto de valores de $\mathbf{x}$ associados aos enlaces de $\mathcal{E}$. Este algoritmo particiona um dado conjunto de vetores em um dado número de classes, cada uma delas representada por um vetor (code-vector) selecionado pelo algoritmo de modo a minimizar o quadrado da distância Euclidiana média entre os vetores de cada classe e o vetor representativo da classe (erro médio quadrático de quantização). Note que quanto menor o erro médio quadrático de quantização desejado, maior o número de classes em que o conjunto de vetores deverá ser particionado. No caso particular em que o erro médio quadrático de quantização desejado é igual a zero, a partição obtida corresponde àquela de classes de enlaces equivalentes.

Como usualmente os valores das densidades de potência de transmissão das diversas emissões presentes no banco de dados da UIT não são caracterizados por um único valor e sim por intervalos de possíveis valores (densidade de potência mínima e densidade de potência máxima), enlaces equivalentes devem possuir o mesmo intervalo $\left[p_{1_{\text {min }}}, p_{1_{\text {max }}}\right]$ de valores de densidade de potência para a transmissão de up-link e o mesmo intervalo $\left[p_{3_{\text {min }}}, p_{3_{\text {max }}}\right]$ de valores de densidade de potência para a transmissão de down-link. Nestes casos, a classificação dos enlaces deve ser baseada em um vetor $\mathbf{x}$ com um número maior de elementos, dado por

$$
\mathbf{x}=\left(\begin{array}{c}
p_{1_{\text {min }}} g_{1}(0) \\
p_{3_{\min }} \\
g_{4}(0) \\
(C / N)_{r e q} \\
\Delta p_{1} \\
\Delta p_{3}
\end{array}\right)
$$

onde

$$
\Delta p_{1}=p_{1_{\max }}-p_{1_{\min }}
$$

e

$$
\Delta p_{3}=p_{3 \max }-p_{3_{\min }}
$$

Nos diagnósticos de utilização da órbita de satélites geoestacionários apresentados nas seções 4.2 e 4.3 a classificação dos enlaces foi feita utilizando-se (A.4). Além disso, a partição em classes de enlaces equivalentes foi suficiente para reduzir o tempo de computação a níveis aceitáveis, não sendo necessária uma redução adicional através de partição em classes de enlaces quasi-equivalentes.

\section{REFERÊNCIAS}

[1] Technical Factors Influencing the Efficiency of the Use of the Geostationary-Satellite Orbit by Radiocommunication Satellites Sharing the Same Frequency Bands, CCIR Report 453, Reports of the CCIR, Annex to Volume IV - Part 1 - FixedSatellite Service, 1990.

[2] Kantor, L. Y., Vybor Iskhodnykh Parametrov dlya Planirovaniya Ispolzovaniya Geostatsionarnoi Orbity FSS (Choice of Basic Parameters for the Planning of the use of the Geostationary Orbit for the FSS), Elektrosviaz, No. 8, 1988.
[3] CCIR Report to the Second Session of the World Administrative Radio Conference on the Use of the GeostationarySatellite Orbit and the Planning of the Space Services Utilizing It, Intenational elecommunication Union, Geneva, 1988.

[4] Radio Regulations, International Telecommunication Union, Vol. 2, Geneva 1998.

[5] Linde, Y., Buzo, A. e Gray, R. M., AnAlgorithm for Vector Quantizer Design, IEEE Transactions on Communications, Vol. COM-28, No. 1, January 1980.

[6] Space Radiocommunications Stations, Base de Dados da União Internacional de Telecomunicações - ITU (Edição de setembro de 2001).

[7] Lambert T., An Optimal Algorithm for Realizing a Delaunay Triangulation, Information Processing Letters, Vol. 62(5), pp 245-250, Junho 1977.

[8] J. M. P. Fortes and R. Sampaio-Neto, An Analytical Method for Assessing Interference in an Environment Involving NGSO Satellite Networks, 1998 IEEE/SBT International Telecommunications Symposium, São Paulo, Brazil, August 1998.

[9] J. M. P. Fortes and R. Sampaio-Neto, An Analytical Method for Assessing Interference in Interference Environments Involving NGSO Satellite Networks, International Journal of Satellite Communications, Vol. 17 No. 6, November-December 1999.

[10] Satellite System Characteristics to be Considered in Frequency Sharing Analyses Between GSO and NON-GSO Satellite Systems in the Fixed Satellite Service including Feeder Links for the Mobile-Satellite Service, Recommendation ITU-R S 13282 (year 2000 version).

[11] Radio Regulations, International Telecommunication Union, 2001.

[12] Reference Earth-Station Radiation Pattern for Use in Coordination and Interference Assessment in the Frequency Range from 2 to About $30 \mathrm{GHz}$, Recommendation ITU-R S 465.

[13] Radiation Diagrams for Use as Design Objectives for Antennas of Earth Stations Operating with Geostationary Satellites, Recommendation ITU-R S 580.

[14] CCIR Report to the Second Session of the World Administrative Radio Conference on the Use of the GeostationarySatellite Orbit and the Planning of the Space Services Utilizing It (WARC-ORB(2)), Part I, pp 44, Geneva 1988.

[15] Proposal of Modification of the Recommends 6 of Recommendation ITU-R S.1323: GSO Earth Station Antenna Pattern to be Used for the Calculations of epfd, Document 4-9-11/156, ITU-R Joint Task Group 4-9-11 meeting, July 1998.

José Mauro P. Fortes graduou-se em Engenharia Elétrica (Telecomunicações) em 1973 pela Pontifícia Universidade Católica do Rio de Janeiro (PUC-Rio). Em 1976 obteve, na mesma universidade, o título de Mestre em Ciências de Engenharia Elétrica. Obteve ainda os títulos de MSc e PhD pela Universidade de Stanford, Califórnia - EUA, em 1978 e 1980, respectivamente. Retornou à PUC-Rio em Junho de 1980, onde é atualmente Professor Associado do Departamento de Engenharia Elétrica, lotado no Centro de Estudos em Telecomunicações da universidade. Durante o ano de 1992, enquanto em licença sabática, foi pesquisador do General Electric Research and Development Center, em Schenectady EUA, atuando no Ultrasound Research Group. O Professor Fortes publicou vários artigos em periódicos e conferências nacionais e internacionais. Participou de diversos projetos de pesquisa e prestou consultoria a diversas empresas privadas e agências governamentais. Durante 13 anos foi Vice-Presidente do Grupo de Estudos 4 (Serviço Fixo por Satélite) do Setor de Radicomunicações da União Internacional de Telecomunicações (ITU), em Genebra. Foi ainda Presidente da Sociedade Brasileira de Telecomunicações de Março de 1996 a Fevereiro de 2000, sendo atualmente Sócio Sênior da 
sociedade. Seus interesses de pesquisa incluem transmissão via satélite, teoria das comunicações, teoria de estimação e transmissão digital.

Raimundo Sampaio Neto Raimundo Sampaio Neto recebeu o diploma de Engenharia Elétrica e o título de Mestre em Engenharia Elétrica pela Pontifícia Universidade Católica no Rio de Janeiro Brasil em 1975 e 1978, respectivamente, e o título de Ph.D. em Engenharia Elétrica pela University of Southern Califórnia (USC), Los Angeles, CA, EUA, em 1983. De 1978 a 1979 foi Professor Assitente na PUC-Rio. De 1979 a 1983 foi estudante de doutorado e pesquisador assitente no Departamento de Engenharia Elétrica da USC, com patrocínio parcial da CAPES. De novembro de 1983 a Junho de 1984 realizou o Pós-Doutorado no Instituto de Ciências em Comunicações do Departamento de Engenharia Elétrica da USC, e foi pesquisador da Axiomatic Corporation, EUA. Durante o ano de 1991, em licença sabática, foi professor visitante do Departamento de Engenharia Elétrica da USC onde realizou seu segundo Pós-dou- torado em Engenharia Elétrica. Desde 1984 até a presente data é pesquisador do Centro de Telecomunicações e professor associado do Departamento de Engenharia Elétrica da PUC-Rio. Atualmente é bolsista em produtividade do $\mathrm{CNPq}$ e suas áreas de interesse incluem teoria das comunicações, sistemas de transmissão digital, comunicações via satélite e detecção multiusuário. Participou de vários projetos e prestou consultoria em telecomunicações para empresas privadas e para o governo brasileiro. Em 1992 foi coorganizador da Seção de Resultados Recentes no Workshop em Teoria da Informação realizado em Salvador e em 1999 foi um dos coordenadores de programa da IEEE Global Telecommunication Conference (Globecom'99) realizada no Rio de Janeiro. Além disso participou como membro de comitês técnicos de diversas conferências nacionais e internacionais. Foi membro do conselho diretor da Sociedade Brasileira de Cominicações por dois mandatos consecutivos. Atualmente é Sócio Sênior da Sociedade Brasileira de Telecomunicações. 